\title{
Research and Validation of Pipe Acoustic Transmission Mechanism
}

\author{
Sun Fuchao ${ }^{1}$, Pei Xiaohan ${ }^{1}$, Zheng Lichen ${ }^{1}$, Wang Guoqing ${ }^{2}$ and Jia Deli ${ }^{1}$ \\ ${ }^{1}$ China Research institute of oil Exploration and Development (RIPED), Petro China, \\ Beijing 100083, China \\ ${ }^{2}$ Daqing Oilfield Production Technology Institute, PetroChina, Daqing 163000, \\ China \\ jiadeli@hrbust.edu.cn
}

\begin{abstract}
During oil production an acoustic communication mode with downhole oil pipe as channel is one of feasible downhole control method. Based on a mechanism analysis, this paper presents a pipe acoustic transmission mechanism model. The model is derived to obtain a pipe dispersive equation. The simulation of phase velocity, group velocity and dispersion curves of three common oil pipes indicates that spectral distribution of longitudinal wave transmission in pipe presents a comb filter structure, and the passband/stopband distribution approximates periodicity and symmetry on spectrogram. The passband range, stopband range and amplitude value changes with length, cross area, density of oil pipe and joint. And the influence rule is given. Then the optimal frequenc. Ds suggested through simulation for downhole wireless communication tools design.
\end{abstract}

Keywords: sound wave; (oil) pipe dispersion; group velocity; comb filter

\section{Introduction}

In order to realize downhole control in oil production engineering sector, electric cable and pressure wave are commonly used for signal transmission. However, these methods need a lot of associated eqaipment, and haye considerable inconvenience due to sophisticated manipulation, ong operation time and high cost. With respect to complex well patterns (e.g., highly-deviated well and horizontal well), cables are hardly run into the planned horizons and thereby unable to achieve downhole control. An oil or water well in the field comprises a downhole pipe string. Therefore, it is optional that surface signals are transmitted downhole by using oil pipe as channel and sound wave as medium. Global scholars have investigated sound wave propagation in drill strings[1-4], but no systematic and profound researches have been conducted on downhole signal transmission in oil/water wells. In this paper, based on one-dimensional wave equation, a pipe acoustic transmission mechanism model is established, and then derived and solved to obtain a dispersive equation. Accordingly, a computer simulation is made. Finally, a set of surface experiment device is prepared to validate the mechanism model.

\section{Establishment of Pipe Acoustic Transmission Mechanism Model}

Sound wave propagation in downhole oil pipes can be described using differential equation of motion, which is constructed according to classic mechanical principles. Wave equation construction is illustrated below through the derivation of longitudinal wave equation in onedimensional string. 
Figure 1 shows the longitudinal vibration and force of sound waves in one-dimensional string. Suppose periodic string has an uniform and infinite structure, cross-section area is $A$, Young's module is $E$, and bulk density is $\gamma$. When oil pipe vibrates longitudinally, each interface keeps flat and round, and cross-section stress distributes evenly.

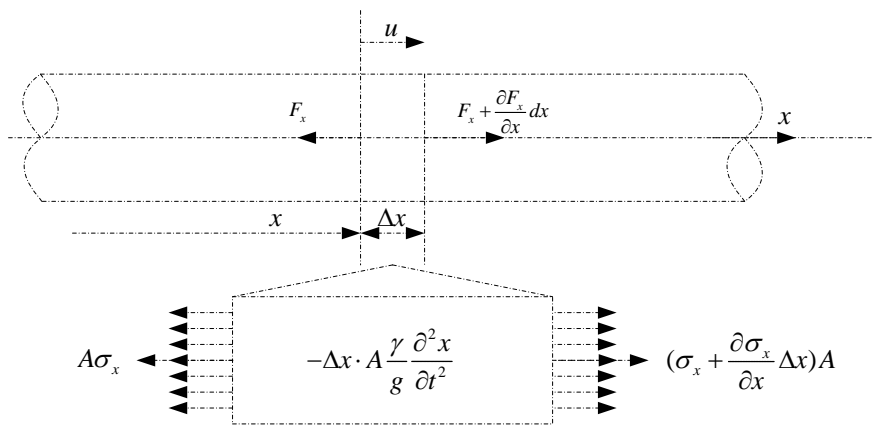

Figure 1. Longitudinal Wave Transmission in One-dimensional String

Motion displacement $(u)$ is a function of pipe axis coordinate $(x)$ and time $(t)$. It is expressed as:

$$
\text { C } u=u(x, t)
$$

In case of elastic wave, the stress-strain relationship for string unit area can be expressed as:

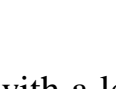

$$
\sigma_{x}=E \frac{\partial u}{\partial x}
$$

A string infinitesimal section with a length of $\Delta x$ is cut out at the point $x$ on the string in Fig. 1, then unit area stres 0 the cross section at the point $(x+\Delta x)$ is $F=\sigma_{x}+\frac{\partial \sigma_{x}}{\partial x} \Delta x$, inertial force imposed on the infinjtesjimal body is $F_{x}=-\Delta x \cdot A \frac{\gamma}{g} \frac{\partial^{2} u}{\partial t^{2}}$, and the following equation is worked out according to Newton's second law:

$$
-\sigma_{x} A+\sigma_{x} A+\frac{\partial \sigma_{x}}{\partial x} \Delta x \cdot A=\Delta x \cdot A \frac{\gamma}{g} \frac{\partial^{2} u}{\partial t^{2}}
$$

It is simplified to:

$$
\frac{\partial \sigma_{x}}{\partial x}=\frac{\gamma}{g} \frac{\partial^{2} u}{\partial t^{2}}
$$

The stress-position relationship can be solved after Eq. (2) is substituted in Eq. (4):

$$
\begin{aligned}
& \frac{\partial^{2} u}{\partial t^{2}}=c^{2} \frac{\partial^{2} u}{\partial x^{2}} \\
& c^{2}=\frac{E}{\rho} \rho=\frac{\gamma}{g}
\end{aligned}
$$

Where, $\rho$ is mass density of medium in string, and $c$ is longitudinal wave velocity in the string. 
Another common description mode of string model is mass coordinate description. Mass coordinate is defined as:

$$
m=\int_{0}^{x} \rho(\xi) a(\xi) d \zeta
$$

The stress-acceleration expression is changed following coordinate changes:

$$
\begin{gathered}
F=z^{2} \frac{\partial u}{\partial m} \\
\frac{\partial u}{\partial t}=\frac{\partial F}{\partial m}
\end{gathered}
$$

Where, impedance $z=\rho a c$.

In this case, two equavilent forms can be obtained after Eqs. (8) and (9 are substituted into Eq. (4). They denote the relationship among displacement, acting force and $\mathrm{ma}$

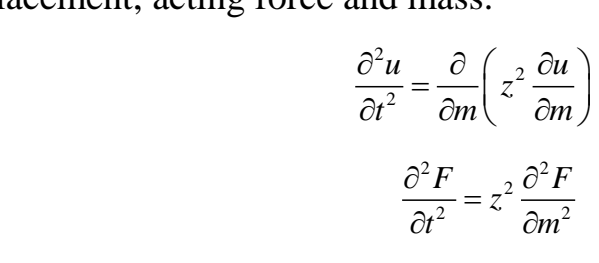

Eqs. (10) and (11) are typical wave equations in mass coordinate, and also basis for analyzing longitudinal wave frequency distribution of the periodic pipes.

\section{Model Solution and Simulation Analysis}

\section{Model Solution}

Phase velocity and group velocity are important parameters describing acoustic transmission properties Phase velocity $\left(c_{p}\right)$ means the velocity at which the phase propagates forward. in is defined as.

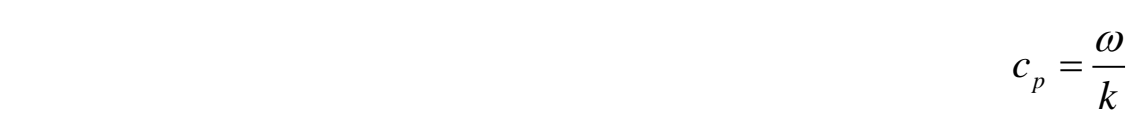

Where, $k$ is wave number, i.e. number of complete waves within a $2 \pi$ unit length.

Generally speaking, wave frequency changes will cause phase velocity changes, which is called dispersion phenomenon. Meanwhile, real transmission of wave energy will be described with group velocity instead of phase velocity:

$$
c_{g}=\frac{d \omega}{d k}
$$

Downhole pipe string is a periodic string structure consisting of oil pipe and coupling, as shown in Figure 2. A periodic string has 2 structures: oil pipe and joint. Its characteristic parameters are constant to be distinguished with subscript $(\xi)$. That is, the subscripts $(\xi)$ of oil pipe and joint are 1 and 2 separately. Each structure has a length of $d_{\xi}$ and a mass of $r_{\xi}$, and

$$
r_{\xi}=\rho_{\xi} a_{\xi} d_{\xi}
$$


If periodic length of a periodic string is expressed as $\mathrm{d}$ in length coordinate $(\mathrm{x})$ and $\mathrm{r}$ in mass coordinate $(\mathrm{m})$, it turns out:

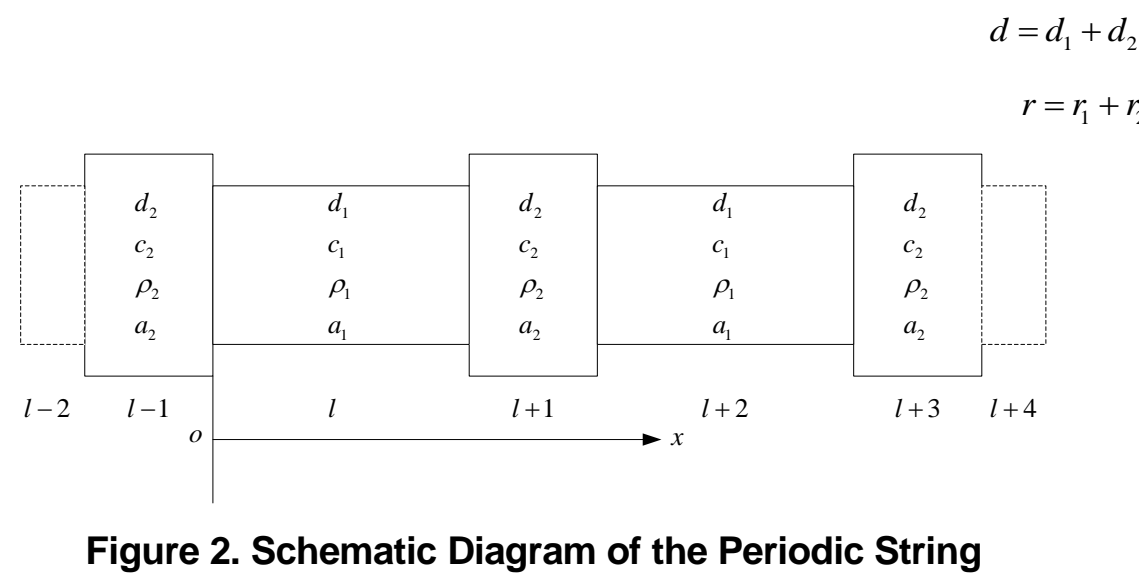

If mass coordinate wave equation is treated by Fournier transform, Fourner Eq. (17) of Eq. (11) can be obtained according to zero initial condition and Fourier differential properties:

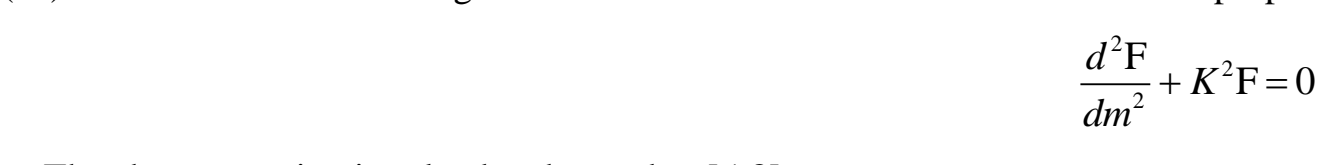

The above equation is solved and sorted to [5-8]:

$$
\cos k d=\cos \left(\frac{\omega d_{1}}{c_{1}} \cos \left(\frac{\omega d_{2}}{c_{2}}\right)-\frac{1}{2}\left(\frac{z_{1}}{z_{2}}+\frac{z_{2}}{z_{1}}\right) \sin \left(\frac{\omega d_{1}}{c_{1}}\right) \sin \left(\frac{\omega d_{2}}{c_{2}}\right)\right.
$$

When sound wave propagates in disuniform periodic pipe, $z_{1} \neq z_{2}$, and wave dispersion phenomenon occurs. Solation of Eq. (18) is a complex number and correlated with frequency.

2. Model Simulation Analysis

If the right part of Eq.(18) lies between -1 and $1,-1<|\cos (k d)|<1$, $\mathrm{k}$ will be real number and there is no attenuation. Instead, $\mathrm{k}$ will attenuate with exponent form if $|\cos (k d)|\rangle 1$. Therefore passband is the frequency band when $\mathrm{k}$ is real and stopband is the frequency band when $\mathrm{k}$ is plural. Phase velocity $\omega / k$ is determined by frequency $\omega$ in passband. So dispersion phenomenon occurr during wave transmission in passband frequencies. According to Equ.(18), frequency structures are influenced by the length, area and density of oil pipe and coupling.

In order to further understand the influences of different periodic string structures on dispersion characteristics, sound wave properties for the three oil pipes are simulated and compared. Here we refer to three pipe sizes commonly adopted in the fields (Table 1), the dispersion equation is solved at wave velocity of $5.05 \times 10^{3} \mathrm{~m} / \mathrm{s}$. 
Table 1. Parameters of Three Oil Pipes

\begin{tabular}{c|c|c|c}
\hline No. of oil pipe & 1 & 2 & 3 \\
\hline OD $(\mathrm{mm})$ & 88.9 & 60.3 & 48.26 \\
\hline ID $(\mathrm{mm})$ & 77.9 & 51.8 & 41.9 \\
\hline Length of oil pipe $(\mathrm{m})$ & $9.76 \sim 9.98$ & $9.75 \sim 9.97$ & $9.83-10 \sim 10$ \\
\hline Cross section area $\left(\mathrm{m}^{2}\right)$ & 0.0062 & 0.0029 & 0.0018 \\
\hline OD of coupling $(\mathrm{mm})$ & 107.95 & 73.03 & 55.88 \\
\hline Length of coupling $(\mathrm{mm})$ & $100-114$ & $114-127$ & $159-178$ \\
\hline Cross section area of coupling & 0.0092 & 0.0042 & 0.0025 \\
\hline
\end{tabular}

Simulation results: seen from the dispersion equation, the frequencies on the right side of the equation with absolute values smaller than 1 will make up the passband; otherwise, they will make up the stopband. Figure 3 gives $\cos k d$ values in relation to frequency. When frequency absolute value is smaller than 1, the corresponding band is passband; when frequency absolute value is bigger than 1, the corresponding bandis stopband. Here we make the frequency range $0-5 \mathrm{kHz}$.

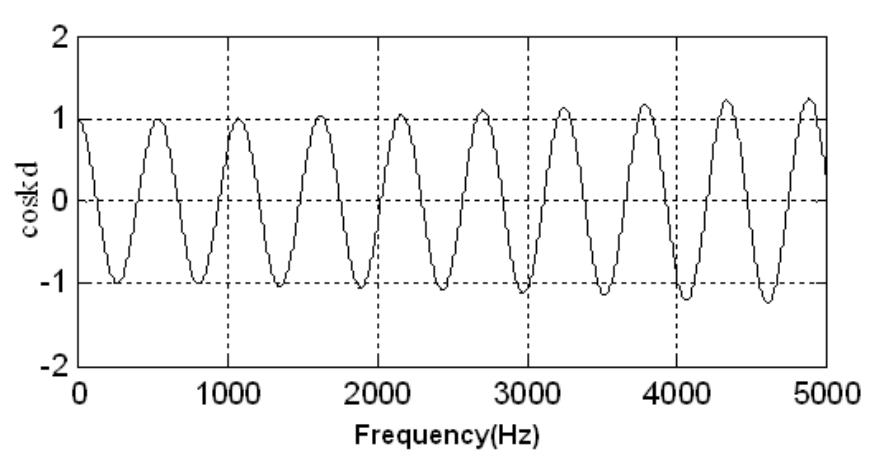

\section{Rigure 3. Curve of $f \sim \cos k d$ in Pipe}

Figure 4 shows the dispersion urye through calculations. It can be seen that periodic string has dispersion phenomenon oceurred due to structural uniqueness, presenting as discontinuous curye. Accôrdug to calculation results in the figure, it is known that passband width decreases and stopband width increases with frequency. Now, $\omega / k$ is no longer a constant. That means longitudinal wave phase velocity for each frequency in the passbands is correlated with frequency.
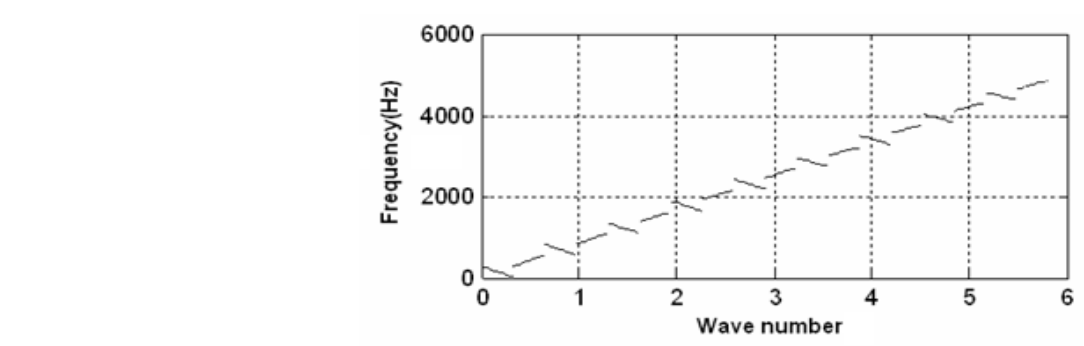

Figure 4. Curve of Wave Number $k$ and Frequency $f$

Figure 5 indicates spectrum characteristics in pipes reflect a comb filter structure with alternating passband and stopband. In the passband, phase and energy transmission velocity 
of each longitudinal wave are no longer constant, but functions of frequency since dispersion phenomenon occurs.

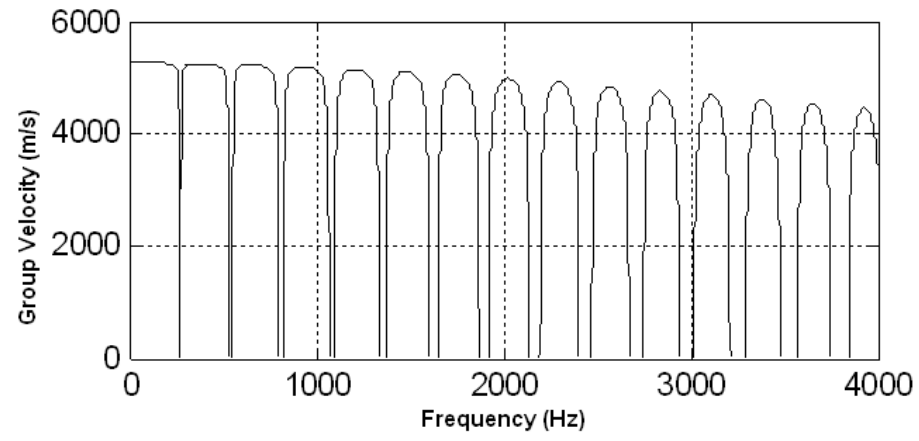

Figure 5. Group Velocity and Frequency Curve in Pipeline

Analysis shows that passband frequency range decreases gradually below $10 \mathrm{KHz}$ through the simulation of the above three oil pipes. This study only sampled $0.5 \mathrm{KH} z$ data for display. Figure 6 reveals $f \sim \cos k d$ curves of three pipes in different sizes.
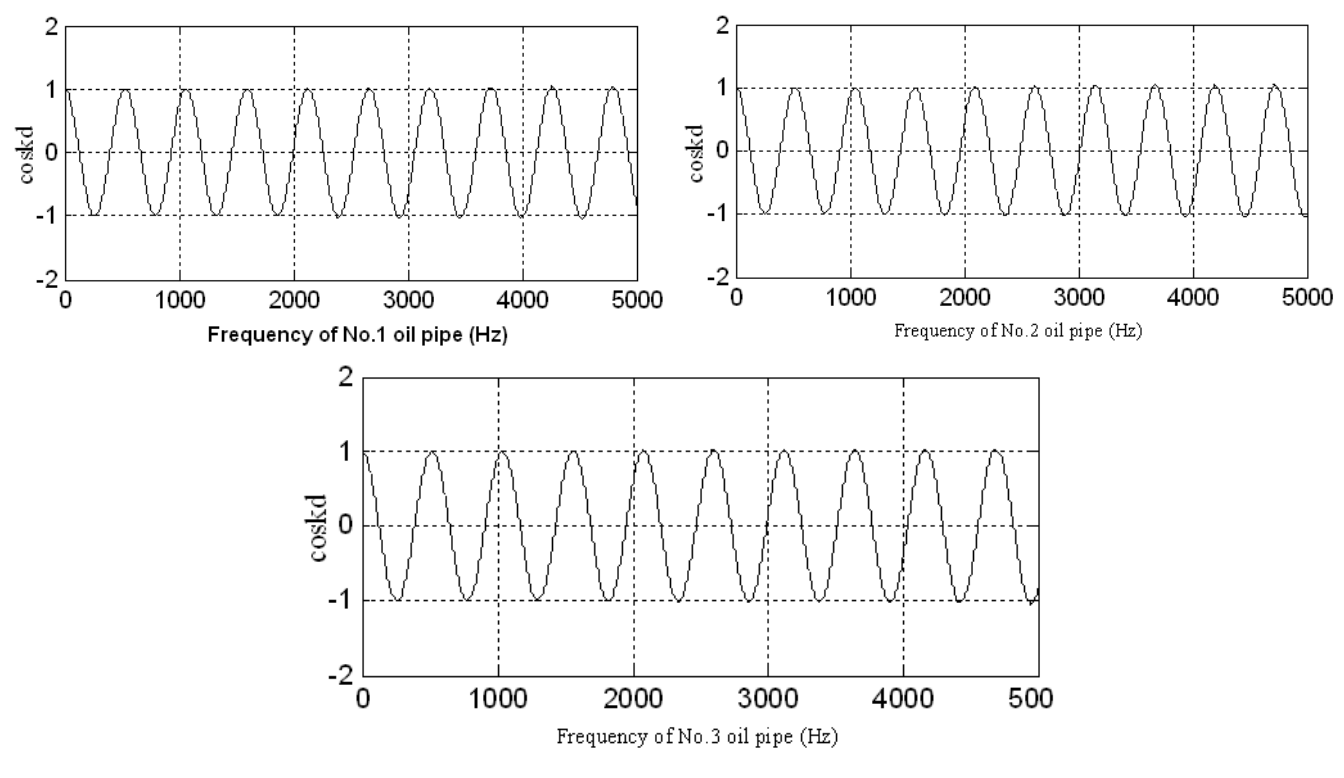

\section{igure 6. $f \sim \cos k d$ Curves of Three Pipes in Different Sizes}

Different pipe sizes influences the waveform transmission in pipe. The wave was compressed $100 \mathrm{~Hz}$ forward of No.3 pipe compared with No.1. The movement of frequency has different effect. The same effect occurs in the parameters of frequency and wave number which is show in Figure 7. 

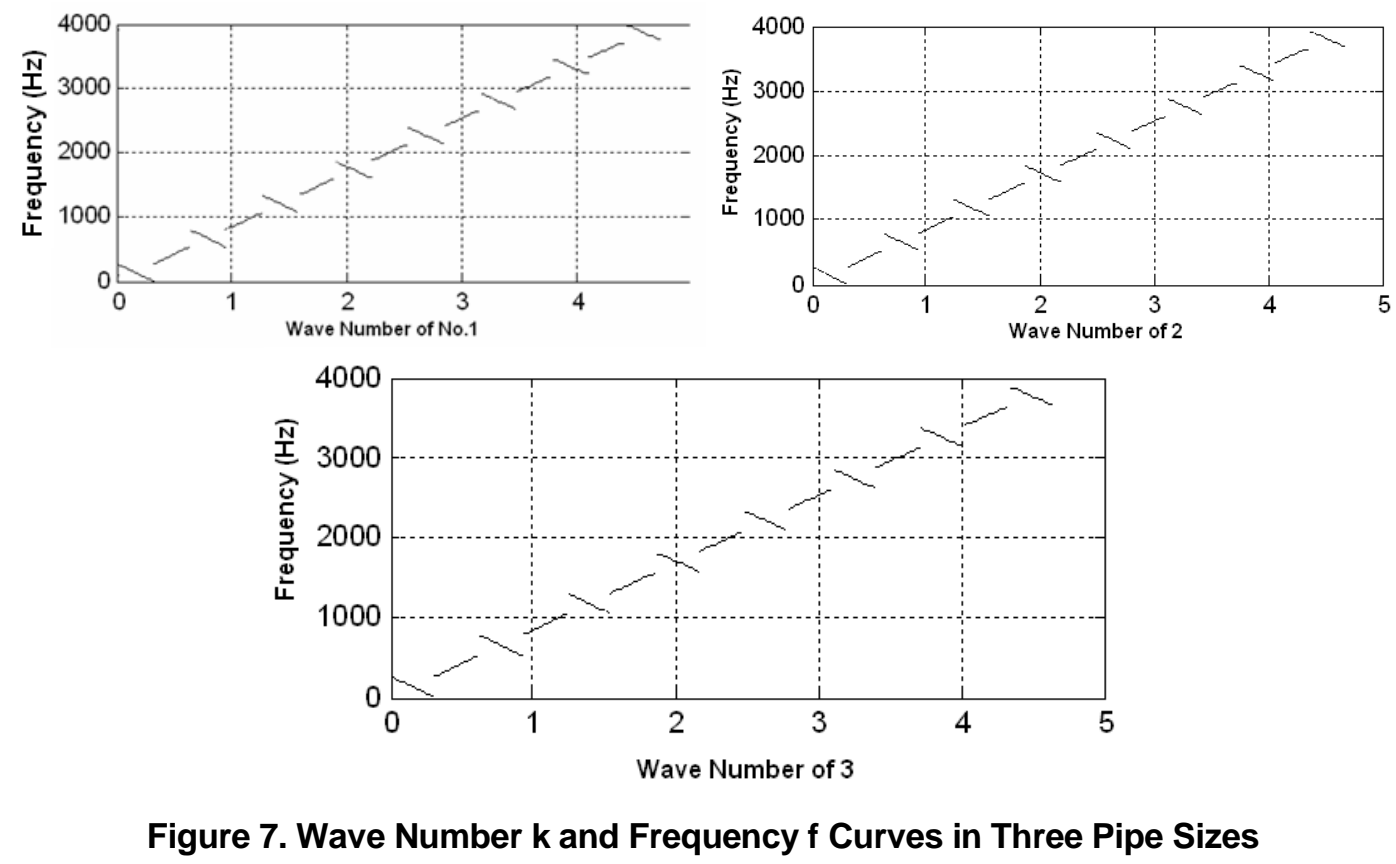

Figure 7. Wave Number $k$ and Frequency $f$ Curves in Thee Pipe Sizes

The group velocity dispersion curves of these oil pipes are constructed accordingly (see Fig. 8). The curves denote that, as frequency increases the comb filter has an overall band changes: passbands get narrower and stopbands become wider progressively. Length increase and cross section decrease of the pipes will make sound wave spectrum distribute with behaviors of simultaneous narrowing and advaneing of the passbands.

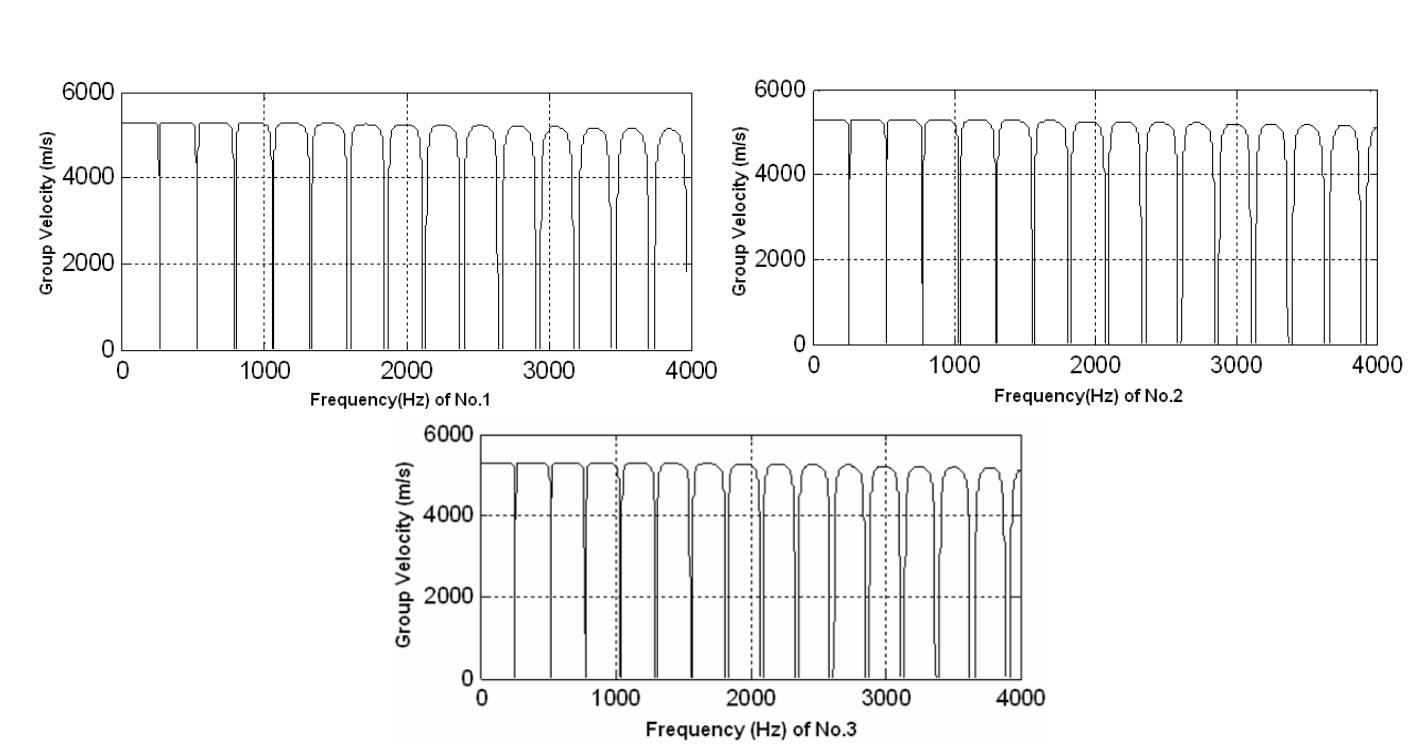

Figure 8. Group Velocity and Frequency Curves in There Pipe Sizes

From the analysis from Figure 6 to Figure 8, we can see that phase velocity and group velocity attenuate a little in passband of wave propagation in pipe. It is benefit for long way transmission taken acoustic wave as carrier. The period length may vary duo to different sizes of pipes. That is to say, no complete period structure will display. Here statistics of passbands 
frequency distributions in relation to three pipe sizes are given in Table 2.Passbandfrequency range is very important for downhole communication tools design, long distance transmission can only be realized when the selected frequency lies in passband range. On the contrary, the signal can not propagate a long distance. So the statistics of passbands frequency distributions may provide a reference for the choice of optimum frequency.

Table 2. Statistics of Passbands Frequency Distributions in Pipe (Hz)

\begin{tabular}{c|c|c|c}
\hline SN & No.1 oil pipe & No.2 oil pipe & No.3 oil pipe \\
\hline 1 & $0-260$ & $0-258$ & $0-256$ \\
\hline 2 & $270-515$ & $275-520$ & $266-512$ \\
\hline 3 & $535-790$ & $545-780$ & $529-770$ \\
\hline 4 & $810-1055$ & $812-1048$ & $792-1028$ \\
\hline 5 & $1075-1320$ & $1080-1312$ & $1055-1286$ \\
\hline 6 & $1345-1587$ & $1347-1577$ & $1317-1545$ \\
\hline 7 & $1613-1852$ & $1615-1841$ & $1579-1804$ \\
\hline 8 & $1882-2116$ & $1884-2105$ & $1840-2064$ \\
\hline 9 & $2151-2380$ & $2153-2369$ & $2102-2323$ \\
\hline 10 & $2419-2646$ & $2421-2632$ & $2363-2583$ \\
\hline 11 & $2688-2911$ & $2690-2896$ & $2625-2842$ \\
\hline 12 & $2956-3176$ & $2959-3160$ & $2888-3103$ \\
\hline 13 & $3225-3411$ & $3227-3424$ & $3150-3359$ \\
\hline 14 & $3493-3706$ & $3496-3689$ & $3413-3618$ \\
\hline 15 & $3762-3970$ & $3764-3954$ & $3675-3877$ \\
\hline
\end{tabular}

\section{Conclusion}

In this paper, based on longitudinal waye equation of one-dimensional string, the pipe acoustic transmission mechanism nodel is established, and then solved to obtain the pipe dispersion equation. By simulation of phase velocity, group velocity and dispersion curves, it is known that the spectraldistribution of longitudinal waves in the pipes present a comb filter structure, and spectral distribution of passbands and stopbands denotes similar features of periodicity and symmetry. The passband has the same change rules with stopband. The passband becomes narrow and stopband becomes broaden with shorter and lower area pipe. Then the optimal frequency of common oil pipes is suggested through simulation for downhole wireless communication tools design. The results and analysis in this research provide a certain theoretral basis for downhole communication in the oilfields.

\section{Acknowledgement}

This work is supported by National High Technology Research and Development Program (863 Program), China (2012AA061302).

\section{References}

[1] L. Brillouin, "Wave propagation in periodic structures", Dover Publications, Inc.,(1953).

[2] T. G. Barnes and B. R. Kirkwood, "Passbands for acoustic transmission in an idealized drill strings", J. Acoust. Soc. Am., vol. 51, (1972), pp. 1606-1608.

[3] J. M. Carcione and F. Poletto, "Simulation of stress waves in attenuating drill strings, including piezoelectric sources and sensors", J. Acoust. Soc. Am.,vol. 108, no. 1, (2000), pp. 53-54.

[4] T. G. Barnes and B. R. Kirkwood, "Passbands for Acoustic Transmission in an Idealized Drill String", J. Acoust. Soc. Amer., vol. 5, (1972), pp. 1606-1608.

[5] D. S. Drumheller, "Attenuation of sound waves in drill strings”, J. Acoust. Soc. Am.,vol. 94, no. 4, (1993), pp. 2387-2396. 
[6] D. S. Drumheller, "The propagation of sound waves in drill strings", J. Acoust. Soc. Am., vol. 97, no. 4, (1995), pp. 2116-2125.

[7] D. S. Drumheller, "Wave impedances of drill strings and other periodic media", J. Acoust.Soc.Am., vol. 112, no. 6, (2002), pp. 2527-2531.

[8] D. S. Drumheller, “Acoustical properties of drill strings", J. Acoust. Soc. Am., vol. 85, (1989), pp. 10481064.

[9] M. Reich, M. Namuq and N. Fischer, "Sounds aus dem rohr Effekte der hydraulischen datenuebertragung in bohrloechern hoerbar gemacht”,Proc. DGMK-Fruehjahrstagung, (2012); Celle.

[10] L. Gao, W. Gardner, C. Robbins, M.Memarzadeh and D. Johnson, "Limits on data communication along the drillstring using acoustic waves", Society of Petroleum Engineers Reservoir Evaluation \&Engi-neering, (2008).

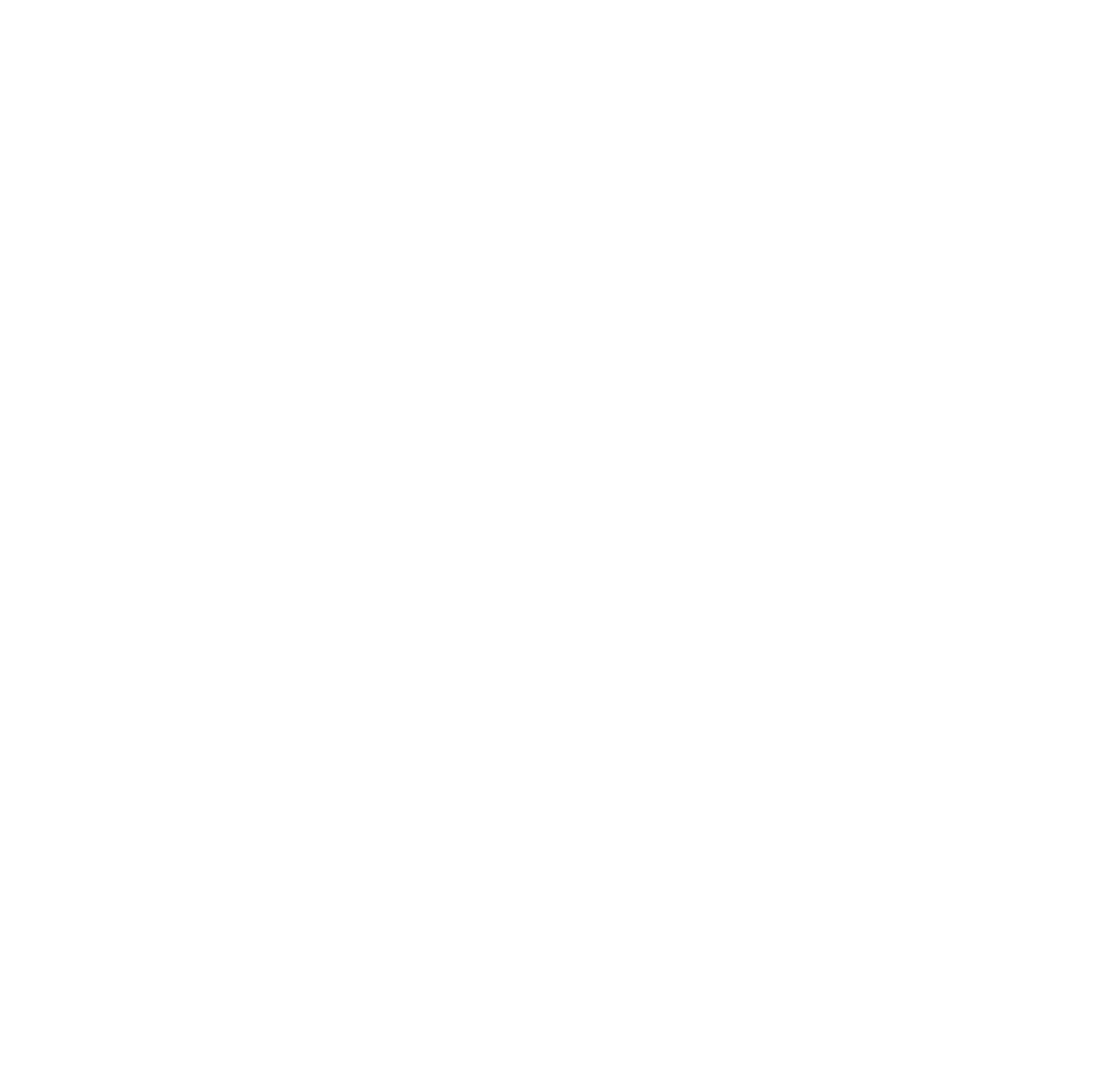


International Journal of Multimedia and Ubiquitous Engineering Vol. 9, No. 11 (2014)

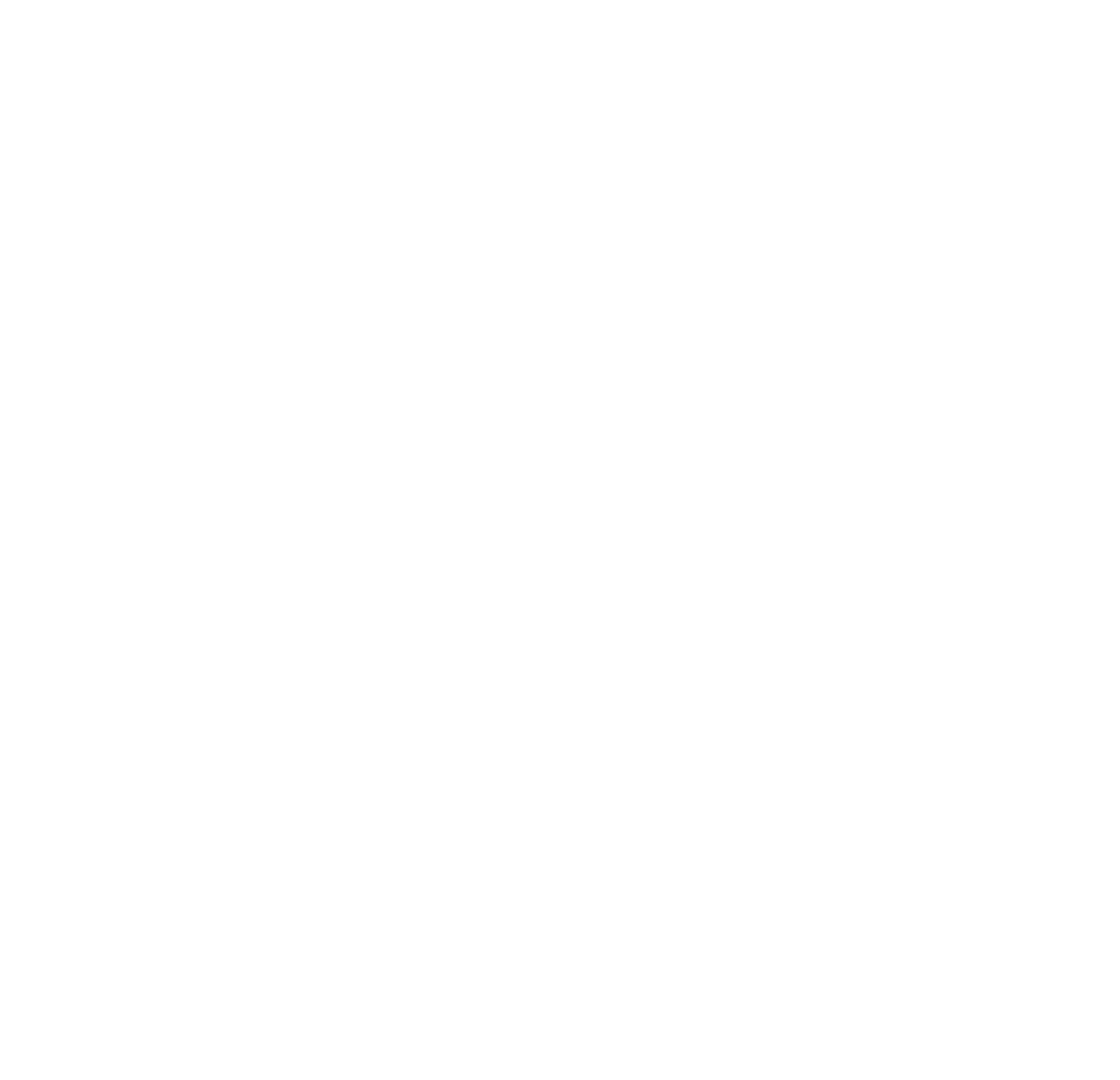

